\title{
Long noncoding RNA EZR-AS1 promotes tumor growth and metastasis by modulating Wnt/ $\beta$-catenin pathway in breast cancer
}

\author{
YU BAI, XIAN ZHOU, LUO HUANG, YUE WAN, XIAOYU LI and YING WANG \\ Department of Radiation Oncology, Chongqing University Cancer Hospital and \\ Chongqing Cancer Institute and Hospital, Chongqing 400030, P.R. China
}

Received February 12, 2018; Accepted May 25, 2018

DOI: $10.3892 /$ etm.2018.6461

\begin{abstract}
Accumulating evidence has demonstrated that long noncoding RNAs (lncRNAs) serve important roles in tumor development and progression. However, whether lncRNA EZR-AS1 is associated with breast cancer (BC) progression remains unclear. In the present study, reverse transcription-quantitative polymerase chain reaction analysis demonstrated that the expression of EZR-AS1 was significantly upregulated in BC tissues and cell lines. Furthermore, Kaplan-Meier curve analysis revealed that increased EZR-AS1 expression in patients with BC contributes to poor prognosis. Cell counting kit- 8 and fluorescence-activated cell sorting experiments indicated that EZR-AS1 knockdown significantly suppressed the proliferation and cell cycle progression of breast cancer cells, while reducing cellular apoptosis. Furthermore, Transwell assays suggested that EZR-AS1 knockdown reduced the migration and invasion ability of BC cells compared with control cells. In the present study, it was observed that EZR-AS1 interacts with $\beta$-catenin to prevent degradation. EZR-AS1 knockdown resulted in $\beta$-catenin downregulation and inactivation of the $\mathrm{Wnt} / \beta$-catenin pathway. Rescue assays revealed that $\beta$-catenin overexpression reversed the effects of EZR-AS1 knockdown on BC cell proliferation, apoptosis, migration and invasion. In conclusion, the results of the present study demonstrate that EZR-AS1 serves as an oncogene in BC via activating the $\mathrm{Wnt} / \beta$-catenin pathway. This suggests that EZR-AS1 may be a therapeutic target for BC treatment.
\end{abstract}

\section{Introduction}

Breast cancer $(\mathrm{BC})$ is one of the most common and malignant cancers affecting women worldwide (1). The incidence of BC

Correspondence to: Dr Ying Wang, Department of Radiation Oncology, Chongqing University Cancer Hospital and Chongqing Cancer Institute and Hospital, 181 Hanyu Road, Shapingba, Chongqing 400030, P.R. China

E-mail: wy2116211@163.com

Key words: EZR-AS1, breast cancer, proliferation, migration, $\beta$-catenin is increasing rapidly, especially in China (2). At present, the main therapeutic regimes for BC treatment include surgery, chemotherapy, radiotherapy and hormonotherapy. However, the treatment outcomes are unsatisfactory (3), due in part to the late diagnosis of $\mathrm{BC}$ (4). The 5-year survival rate of $\mathrm{BC}$ patients is very low (5). As such, there is an urgent need to identify novel biomarkers to aid in the diagnosis and treatment of BC.

Long noncoding RNAs (lncRNAs) are a class of noncoding RNAs $>200$ nucleotides in length (6). Over the past decade, lncRNAs have been the subject of a great deal of research (7). A number of studies have indicated that lncRNAs serve important roles in many biological processes, including development, immune regulation and cancer development (8-10). A number of studies have demonstrated that lncRNAs are able to regulate the proliferation, apoptosis, migration and invasion of human cancer cells $(11,12)$. Wang et al (13) reported that HOXD-AS1 promotes cell proliferation, migration and invasion via the microRNA (miR)-608/FZD4 axis in ovarian cancer. Pan et al (14) demonstrated that lncRNA Differentiation Antagonizing Non-Protein Coding RNA is activated by sal-like protein 4 and promotes the proliferation and invasion of gastric cancer cells. Aberrant expression of lncRNAs is typically observed in human cancers (10) and several lncRNAs have been identified as potential diagnostic and prognostic biomarkers in cancer (15). Therefore, elucidating the function and mechanism of lncRNAs is crucial for understanding the pathogenesis of human cancers.

A previous study indicated that EZR-AS1 promotes the migration and invasion of human esophageal squamous cell carcinoma cells (16). However, the function of EZR-AS1 in breast cancer remains largely unknown. The current study aimed to investigate the function and mechanism of EZR-AS1 in breast cancer progression. Based on cell counting kit (CCK)-8, fluorescence-activated cell sorting (FACS) and Transwell experiments, it was demonstrated that EZR-AS1 may serve as an oncogene by activating the Wnt/ $\beta$-catenin pathway in breast cancer.

\section{Materials and methods}

Patient samples. A total of 37 breast cancer tissues and 13 adjacent normal tissues were collected from patients, aged $53 \pm 12$ years, undergoing resection surgery at Chongqing University Cancer Hospital \& Chongqing Cancer Institute \& 
Hospital (Chongqing,China) between January 2014 to December 2016. Tissue specimens were flash frozen and stored in liquid nitrogen until further use. No patients received preoperative chemotherapy or radiation and patients with hypertension or diabetes mellitus were excluded from the present study. This study was approved by the Ethics and Research Committees of Chongqing University Cancer Hospital \& Chongqing Cancer Institute \& Hospital and was performed in accordance with the Declaration of Helsinki. All patients provided written informed consent.

Cell culture and transfection. MCF-10A, a normal breast epithelial cell line and human breast cancer cell lines MCF7, MDA-MB-231, MDA-MB-468 and SKBR-3 were purchased from American Type Culture Collection (Manassas, VA, USA). All cells were cultured in Dulbecco's modified Eagle's medium (DMEM; Gibco; Thermo Fisher Scientific, Inc., Waltham, MA, USA) supplemented with $10 \%$ fetal bovine serum (FBS; Gibco; Thermo Fisher Scientific, Inc.), $50 \mathrm{U} / \mathrm{ml}$ penicillin and $0.1 \mathrm{mg} / \mathrm{ml}$ streptomycin at $37^{\circ} \mathrm{C}$ in an atmosphere containing 5\% $\mathrm{CO}_{2}$. Cycloheximide (Chx; cat. no. 2112; Cell Signaling Technology, Inc., Danvers, MA, USA) was administered at $15 \mu \mathrm{g} / \mathrm{ml}$ and was added to MCF7 cells cultured in 6 -well plates. Following incubation for 0,2 or $4 \mathrm{~h}$ at $37^{\circ} \mathrm{C}$, cells were collected by centrifugation $\left(2,500 \mathrm{x} \mathrm{g}, 5 \mathrm{~min}, 4^{\circ} \mathrm{C}\right)$ and analyzed using western blot.

For cell transfection, EZR-AS1 small interfering (si)RNA (siEZR-AS1) targeting the non-overlapping domain between EZR and EZR-AS1, specifically located within the third exon of EZR-AS1, was synthesized by Shanghai GenePharma Co., Ltd. (Shanghai, China). The sequence of siEZR-AS1 was 5'-UAUUUUCCAAAUCUUUUCC-3'. Scramble shRNA (shNC) was also synthesized by Shanghai GenePharma Co., Ltd. Cells were transfected with $30 \mathrm{nM}$ siRNA using Lipofectamine $^{\circledR} 3000$ (Invitrogen; Thermo Fisher Scientific, Inc.) according to the manufacturer's protocol and harvested at $48 \mathrm{~h}$ post-transfection.

RNA immunoprecipitation (RIP) assay. Untransfected MCF7 or MDA-MB-231 cells $\left(5 \times 10^{6}\right)$ in an open dish on ice were placed in a Stratalinker UV-light box and irradiated at $254 \mathrm{~nm}$ for $2 \mathrm{~min}$. DMEM was removed and $1 \mathrm{ml}$ cold PBS added to each plate. Cells were scraped, collected into tubes and centrifuged at $100 \mathrm{xg}$ for $5 \mathrm{~min}$ at $4^{\circ} \mathrm{C}$. PBS was removed and cells were lysed in cell lysis buffer (5 mM HEPES pH 7.4, $85 \mathrm{mM}$ $\mathrm{KCl}, 0.5 \% \mathrm{NP} 40$ ) for $8 \mathrm{~min}$ at $4^{\circ} \mathrm{C}$ with shaking. Samples were transferred to tubes and centrifuged at 2,500 $\mathrm{x}$ g for $5 \mathrm{~min}$ at $4^{\circ} \mathrm{C}$. Supernatant lysate was precleared by incubation with $40 \mu \mathrm{l}$ protein G-coupled Dynabeads (Thermo Fisher Scientific, Inc.) for $30 \mathrm{~min}$ at $4^{\circ} \mathrm{C}$. Following centrifugation $(2,500 \mathrm{xg}, 5 \mathrm{~min}$, $4^{\circ} \mathrm{C}$ ), the supernatant was collected and Protein G-coupled Dynabeads were discarded. Then $40 \mu 1$ protein G-coupled Dynabeads and $3 \mu \mathrm{g}$ anti- $\beta$-catenin antibody $(1: 1,000$; cat. no. 8480; CST Biological Reagents Co., Ltd.) were added to the supernatant and mixed overnight at $4^{\circ} \mathrm{C}$. Following, beads were collected by centrifugation at 2,500 $\mathrm{xg}$ for $5 \mathrm{~min}$ at $4^{\circ} \mathrm{C}$ and washed $4 \mathrm{x}$ with lysis buffer ( $5 \mathrm{mM}$ HEPES $\mathrm{pH} 7.4,85 \mathrm{mM} \mathrm{KCl}$, $0.5 \%$ NP40). RNA was isolated from the beads using TRIzol reagent (Invitrogen; Thermo Fisher Scientific, Inc.), incubated with DNase I (Sigma Aldrich; Merck KGaA, Darmstadt,
Germany) and subjected to reverse transcription-quantitative polymerase chain reaction (RT-qPCR).

RNA pull-down assay. EZR-AS1 and EZR-AS1 antisense DNA templates containing a T7 promoter were synthesized by Sangon Biotech Co., Ltd. (Shanghai, China). Biotin-labeled EZR-AS1 and EZR-AS1 antisense, which served as a negative control, were obtained by in vitro transcription with a Biotin RNA Labeling mix (Roche Diagnostics, Basel, Switzerland) containing T7 RNA polymerase according to the manufacturer's protocol. Biotin-labeled RNA was treated with RNase-free DNase I (Roche Diagnostics) according to the manufacturer's protocol and purified with an RNeasy Mini kit (Qiagen, Inc., Valencia, CA, USA) according to the manufacturer's instructions. Concentrations were detected using a Chemiluminescent Nucleic Acid Detection module (No. 89880; Thermo Fisher Scientific, Inc.). A total of $20 \mu \mathrm{l}$ biotin-labeled RNA and $40 \mu \mathrm{l}$ streptavidin Dynabeads (Thermo Fisher Scientific, Inc.) were added to $1 \mathrm{ml} \mathrm{MCF7}$ cell supernatant lysate for $6 \mathrm{~h}$ at $25^{\circ} \mathrm{C}$. Beads were isolated from the supernatant $(2,500 \mathrm{x} \mathrm{g}, 5 \mathrm{~min}$, $\left.4^{\circ} \mathrm{C}\right)$ and washed with wash buffer $(10 \mathrm{mM}$ Tris- $\mathrm{HCl} \mathrm{pH} \mathrm{7.5,}$ $1 \mathrm{mM}$ EDTA, $2 \mathrm{M} \mathrm{NaCl}$ and $0.1 \%$ Tween-20) followed by a centrifugation step $\left(2,500 \times \mathrm{g}, 5 \mathrm{~min}, 4^{\circ} \mathrm{C}\right)$. Isolated beads were mixed with $20 \mu \mathrm{l}$ SDS loading buffer (Beyotime Institute of Biotechnology, Haimen, China) and boiled for $10 \mathrm{~min}$ at $100^{\circ} \mathrm{C}$. Precipitated proteins were detected by western blotting.

Western blot analysis. MCF7 or MDA-MB-231 cells $\left(2 \times 10^{6}\right)$ were lysed with $1 \mathrm{ml}$ radio immunoprecipitation assay buffer (Beyotime Institute of Biotechnology) and protein was isolated by centrifugation $\left(13,500 \mathrm{x} \mathrm{g}, 10 \mathrm{~min}, 4^{\circ} \mathrm{C}\right)$ and quantified by bicinchoninic acid analysis (Beyotime Institute of Biotechnology). Protein extracts $(30 \mu \mathrm{g})$ were resolved using $10 \%$ SDS-PAGE gels and transferred to polyvinyl difluoride membranes (EMD Millipore, Billerica, MA, USA). Following blocking in Tris-buffered saline containing $0.1 \%$ Tween- 20 (TBS-T) with $5 \%$ nonfat dry milk for $30 \mathrm{~min}$ at $37^{\circ} \mathrm{C}$, membranes were washed four times in TBS-T and incubated with primary antibodies overnight at $4^{\circ} \mathrm{C}$. Primary antibodies were used at the following dilutions: Anti-GAPDH (1:1,000; cat. no. 5174; CST Biological Reagents Co., Ltd., Shanghai, China), anti-cyclin D1 (1:1,1000; cat. no. 2978; CST Biological Reagents Co., Ltd.), anti-MYC (1:1,000; cat. no. 2276; CST Biological Reagents Co., Ltd.), anti-sry box-4 (SOX4; 1:2,000; cat. no. SAB2108306; Sigma Aldrich; Merck KGaA) and anti- $\beta$-catenin (1:1,000; cat. no. 8480; CST Biological Reagents Co., Ltd.). Following washing, membranes were incubated with horseradish peroxidase-linked goat polyclonal anti-rabbit IgG secondary antibody $(1: 2,000$; cat. no. 7074; CST Biological Reagents Co., Ltd.) for $1 \mathrm{~h}$ at room temperature. Immunoreactivity was detected by enhanced chemiluminescence (Thermo Scientific Fisher, Inc.) according to the manufacturer's instructions. GAPDH served as the loading control.

$R T-q P C R$ analysis. Total RNA was extracted from tissues or cultured cells using TRIzol reagent (Invitrogen; Thermo Fisher Scientific, Inc.) according to the manufacturer's instructions and subjected to RT using SuperScript ${ }^{\mathrm{TM}}$ IV Reverse Transcriptase (Thermo Fisher Scientific, Inc.) at $37^{\circ} \mathrm{C}$ for 
$25 \mathrm{~min}$, followed by incubation at $85^{\circ} \mathrm{C}$ for $5 \mathrm{sec}$ in $20 \mu \mathrm{l}$ of reaction volume according to the manufacturer's instructions. qPCR was performed using Trans Start Top Green qPCR Super mix (Beijing TransGen Biotech Co., Ltd., Beijing China). The PCR reaction was evaluated using melting curve analysis. The conditions for PCR reaction were as follows: Initial denaturation at $95^{\circ} \mathrm{C}$ for $10 \mathrm{sec}$, followed by 40 cycles of $95^{\circ} \mathrm{C}$ for $5 \mathrm{sec}, 55^{\circ} \mathrm{C}$ for $30 \mathrm{sec}$ and $72^{\circ} \mathrm{C}$ for $20 \mathrm{sec}$. Results were evaluated using the $2^{-\Delta \Delta \mathrm{Cq}}$ method (17). RNA18SN5 was used to normalize EZR-AS1. The primer sequences were as follows: EZR-AS1 forward 5'-CCCTCTCCAATGAAGCCT CTC-3' and reverse 5'-ACCGAAAATGCCGAAACCAG-3'; MYC forward 5'-CCCTCCACTCGGAAGGACTA-3' and reverse 5'-GCTGGTGCATTTTCGGTTGT-3'; SOX4 forward 5'-GCACTAGGACGTCTGCCTTT-3' and reverse 5'-ACA CGGCATATTGCACAGGA-3'; 18S forward 5'-GTGCCA GCAGCCGCGGTAATTC-3' and reverse 5'-ACACTCAGC TAAGAGCATCG-3'; and GAPDH forward 5'-TCCTCTGAC TTCAACAGCGACAC-3' and reverse 5'-CACCCTGTTGCT GTAGCCAAATTC-3'.

CCK- 8 proliferation assays. Transfected cells were collected at $24 \mathrm{~h}$ post-transfection and seeded into 96 -well plates at a density of $3 \times 10^{3}$ cells/well. Following $0,24,48$ or 72 h culture, a CCK-8 assay was performed according to the manufacturer's protocol In brief, $10 \mu \mathrm{l}$ of CCK-8 reagent (Beyotime Institute of Biotechnology) was added to each well at $37^{\circ} \mathrm{C}$ for $2 \mathrm{~h}$ in an atmosphere containing $5 \% \mathrm{CO}_{2}$. Absorbance was measured at a wavelength of $450 \mathrm{~nm}$ using an ELx808 absorbance reader (BioTek Instruments, Inc., Winooski, VT, USA). Each assay was performed in triplicate and repeated three times.

Migration and invasion assays. For the Transwell migration assay, 24-well inserts with $8 \mathrm{~mm}$ pore size polycarbonate membranes (Corning, Inc., Corning, NY, USA) were used. A total of $700 \mu \mathrm{l}$ DMEM supplemented with $20 \%$ FBS was added to the lower chamber and $1 \times 10^{5}$ cells resuspended in serum-free media were added to the upper insert. Transwell membranes were fixed with $4 \%$ formaldehyde for $2 \mathrm{~h}$ at $25^{\circ} \mathrm{C}$ and stained using $0.5 \%$ crystal violet for $1 \mathrm{~h}$ at $25^{\circ} \mathrm{C}$. Cells adhering to the lower surface of the membrane were counted under a light microscope (Olympus Corp., Tokyo, Japan). For the invasion assay, cells were plated onto BioCoat Matrigel Invasion Chambers (pore size $8 \mu \mathrm{m}$; BD Biosciences, Franklin Lakes, NJ, USA). Other steps were the same as in the migration assay.

Apoptosis assay. Cell apoptosis was assessed using flow cytometry with an Annexin V-fluorescein isothiocyanate/propidium iodide apoptosis detection kit (Nanjing KeyGen Biotech Co., Ltd., Nanjing, China) according to the manufacturer's protocol. A FACSAria flow cytometer (BD Biosciences) was used.

Statistical analysis. Each experiment was repeated at least three times. All data are expressed as the mean \pm standard deviation. The Kaplan-Meier method was used to calculate the survival curve and a log-rank test was performed to determine statistical significance. Pearson's correlation coefficient analysis was used to determine correlations between groups. Student's t-test and one-way analysis of variance followed by Tukey's post hoc test were used for statistical analysis. $\mathrm{P}<0.05$ was considered to indicate a statistically significant difference.

\section{Results}

EZR-AS1 was upregulated in BC tissues. To explore the function of EZR-AS1, expression patterns were assessed using RT-qPCR. It was demonstrated that EZR-AS1 was significantly upregulated in BC tissues compared with adjacent normal tissues (Fig. 1A). The expression of EZR-AS1 was assessed in 13 pairs of $\mathrm{BC}$ and adjacent normal tissues. The results indicated that EZR-AS1 was highly expressed in the majority of BC tissues compared with paired adjacent normal tissues (Fig. 1B). Furthermore, the expression of EZR-AS1 was upregulated in $\mathrm{BC}$ cell lines compared with breast epithelial MCF-10A cells (Fig. 1C). It was next assessed whether EZR-AS1 may be used as a prognostic biomarker for patients with BC. The results of Kaplan-Meier analysis revealed that upregulated EZR-AS1 expression in patients with BC predicted poor prognosis (Fig. 1D). Taken together, these data suggest that EZR-AS1 is upregulated in BC tissues and may serve a role in disease progression.

EZR-AS1 knockdown suppressed BC cell proliferation and induced apoptosis. MCF7 and MDA-MB-231 BC cells were used to investigate the role of EZR-AS1. RT-qPCR revealed that EZR-AS1 was significantly downregulated in MCF7 and MDA-MB-231 cells transfected with si-EZR-AS1 compared with the controls (Fig. 2A). CCK8 assays were performed to assess cell proliferation and the results indicated that EZR-AS1 knockdown significantly inhibited the proliferation of MCF7 and MDA-MB-231 cells compared with control cells (Fig. 2B and $\mathrm{C}$ ). To determine whether reduced proliferation was a result of cell cycle disruption, the effect of EZR-AS1 on the cell cycle was assessed using flow cytometry. It was revealed that EZR-AS1 knockdown significantly reduced the number of MCF7 and MDA-MB-231 cells in S phase compared with the control group (Fig. 2D). Furthermore, EZR-AS1 knockdown promoted the apoptosis of MCF7 and MDA-MB-231 cells compared with control cells (Fig. 2E). These results suggest that EZR-AS1 knockdown suppresses BC cell proliferation and induces apoptosis.

EZR-AS1 knockdown inhibited the migration and invasion of $B C$ cells. Tumor metastasis is often linked to malignance (18). In the present study, the effects of EZR-AS1 on BC cell metastasis were investigated. The results of Transwell and Matrigel assays revealed that EZR-AS1 knockdown significantly suppressed the migration and invasion of MCF7 and MDA-MB-231 cells in vitro (Fig. 3A and B).

EZR-AS1 protected $\beta$-catenin from degradation and activated the Wnt/ $\beta$-catenin pathway in BC cells. The mechanism of EZR-AS1 in BC was assessed and it was demonstrated that anti- $\beta$-catenin enriched endogenous EZR-AS1 in MCF7 and MDA-MB-231 cells (Fig. 4A). Furthermore, biotin-labeled EZR-AS1 precipitated endogenous $\beta$-catenin in MCF7 cells (Fig. 4B). These data suggest that there was an interaction between EZR-AS1 and $\beta$-catenin. Chx administration and 

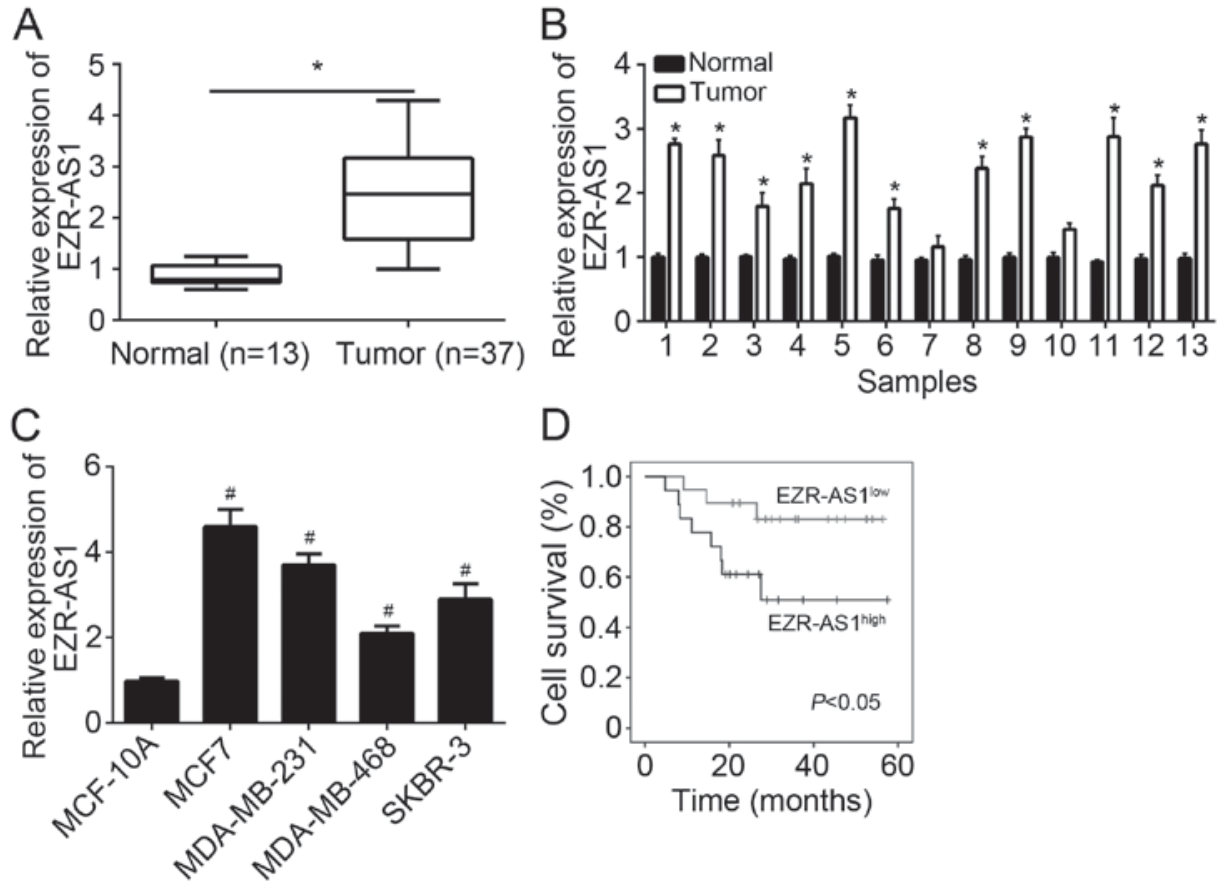

Figure 1. EZR-AS1 was upregulated in BC tissues. Relative expression of EZR-AS1 in (A) 37 BC tissues and 13 adjacent normal tissues, (B) 13 pairs of BC and adjacent normal tissues and (C) BC cell lines and normal MCF-10A cells. (D) A Kaplan-Meier curve was constructed based on EZR-AS1 expression in BC tissues. ${ }^{\text {P }}<0.05$ vs. normal and ${ }^{\#} \mathrm{P}<0.05$ vs. MCF-10A. All data are presented as the mean \pm standard deviation of three independent experiments. BC, breast cancer; RT-qPCR, reverse transcription-quantitative polymerase chain reaction.

A

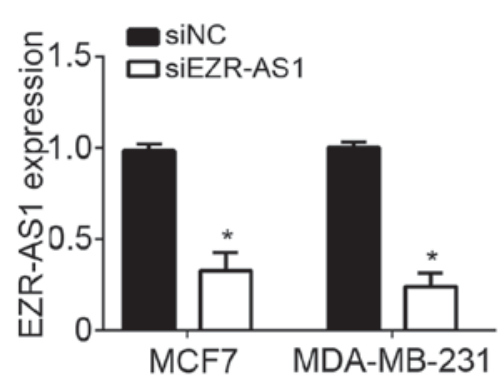

D

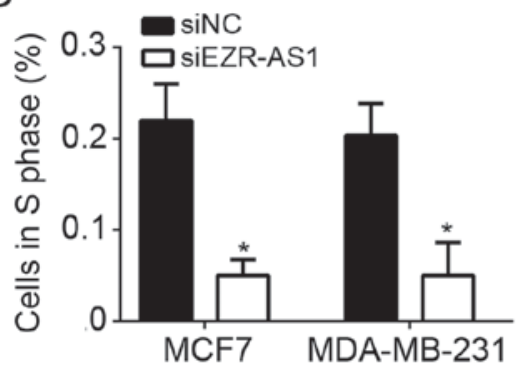

B

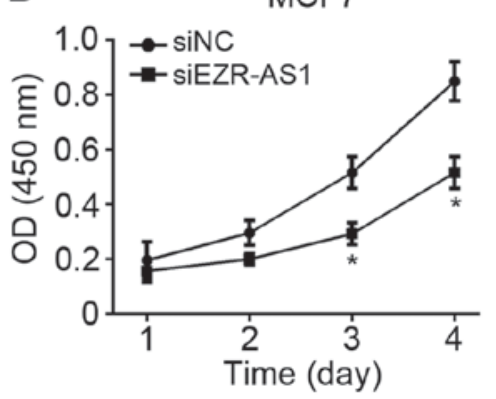

E

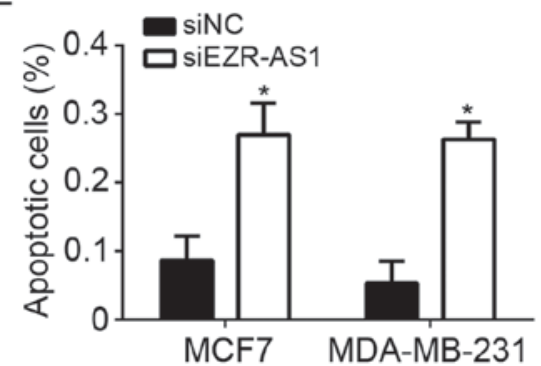

C

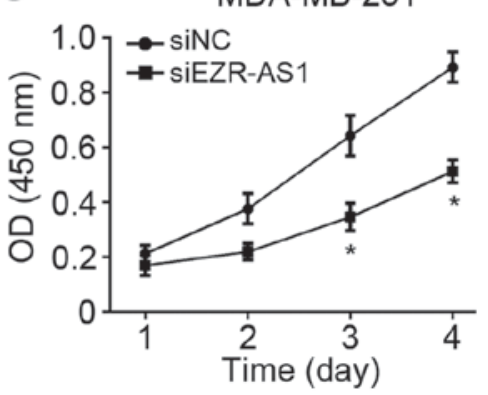

Figure 2. EZR-AS1 knockdown suppresses BC cell proliferation and induces apoptosis. (A) Reverse transcription-quantitative polymerase chain reaction was performed to assess EZR-AS1 expression in MCF7 and MDA-MB-231 cells transfected with sh-NC or sh-EZR-AS1. Cell Counting Kit 8 assays were performed to assess the proliferation of (B) MCF7 and (C) MDA-MB-231 cells. (D) The percentage of (D) cells in S phase and (E) apoptotic MCF7 and MDA-MB-231 cells was assessed using flow cytometry. ${ }^{*} \mathrm{P}<0.05$ vs. siNC. All data are presented as the mean \pm standard deviation of three independent experiments. BC, breast cancer; sh, short hairpin RNA; NC, negative control.

western blot analysis revealed that EZR-AS1 overexpression significantly delayed the degradation of $\beta$-catenin in MCF7 cells compared with the control (Fig. 4C). Western blotting results indicated that EZR-AS1 knockdown suppressed the nuclear expression of $\beta$-catenin, MYC, SOX4 and cyclin D1 in MCF7 and MDA-MB-231 cells compared with control cells (Fig. 4D). Furthermore, it was revealed that the expression of EZR-AS1 was positively correlated with that of MYC or SOX4 in BC tissues (Fig. 4E), suggesting that EZR-AS1 promotes the activation of the Wnt/ $\beta$-catenin pathway. 

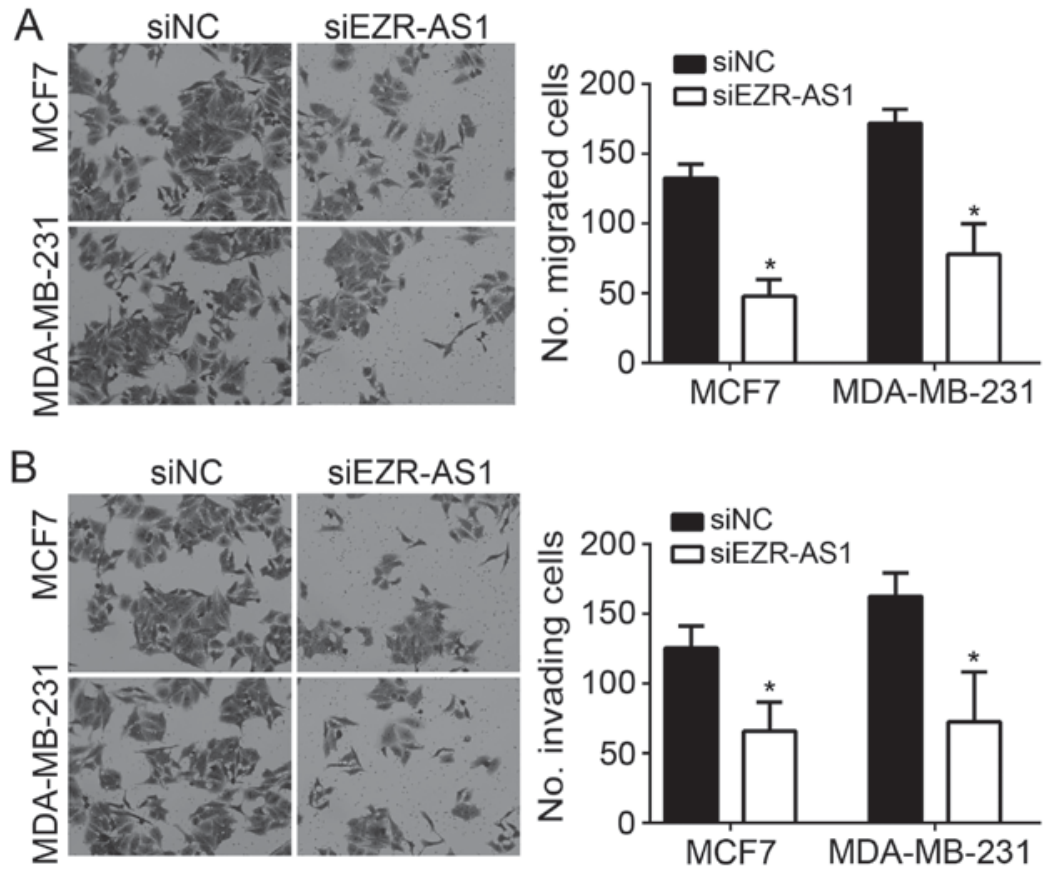

Figure 3. EZR-AS1 knockdown inhibits the migration and invasion of BC cells. Transwell and Matrigel assays were performed to assess the (A) migration and (B) invasion of MCF7 and MDA-MB-231 cells following EZR-AS1 knockdown (light microscope; magnification, x100). " $\mathrm{P}<0.05$ vs. siNC. All data are presented as the mean \pm standard deviation of three independent experiments. si, small interfering RNA; NC, negative control.

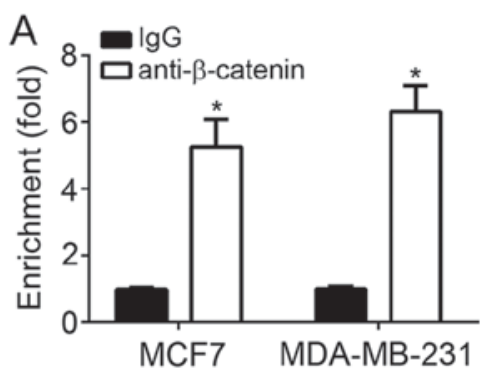

B
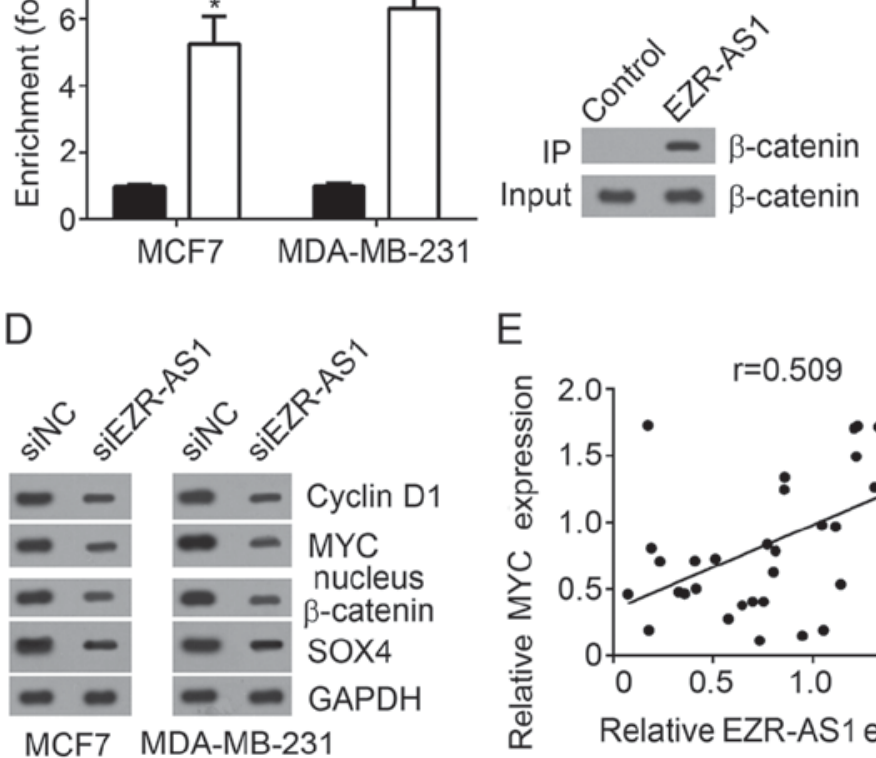

E

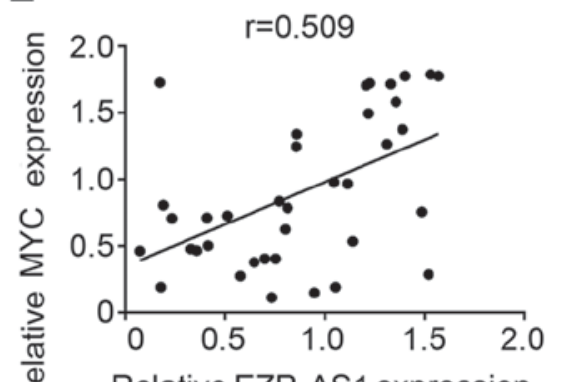

Relative EZR-AS1 expression
C

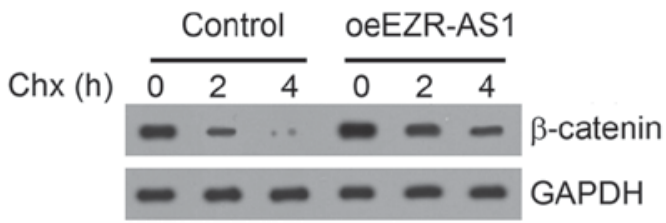

Figure 4. EZR-AS1 protects $\beta$-catenin from degradation and activates the Wnt $/ \beta$-catenin pathway in BC cells. (A) RNA immunoprecipitation assays indicated that EZR-AS1 was enriched by anti- $\beta$-catenin in MCF7 and MDA-MB-231 cells. (B) An RNA pull-down assay indicated that $\beta$-catenin was precipitated by biotin-labeled EZR-AS1. (C) EZR-AS1 overexpression prevented $\beta$-catenin degradation in MCF7 cells. (D) Western blotting was performed to assess the effect of EZR-AS1 knockdown on nuclear $\beta$-catenin, MYC, SOX4 and Cyclin D1 expression in MCF7 and MDA-MB-231 cells. (E) Correlation between EZR-AS1 and MYC or SOX4 in BC tissues. ${ }^{*} \mathrm{P}<0.05 \mathrm{vs.} \mathrm{IgG}$. All data are presented as the mean \pm standard deviation of three independent experiments. BC, breast cancer; SOX, SRY box; oe, overexpression; si, small interfering RNA; NC, negative control; Ig, immunoglobulin.

Restoration of $\beta$-catenin expression abrogated the effects of EZR-AS1 knockdown. To further confirm that $\beta$-catenin is crucial for EZR-AS1 to function in BC cells, the expression of $\beta$-catenin was restored using ectopic expression. Western blotting revealed that EZR-AS1 knockdown significantly inhibited the expression of $\beta$-catenin in MCF7 and MDA-MB-231 cells compared with negative control cells, while ectopic $\beta$-catenin expression restored its levels (Fig. 5A). CCK8 assays were performed to assess cell proliferation and it was demonstrated that EZR-AS1 
A
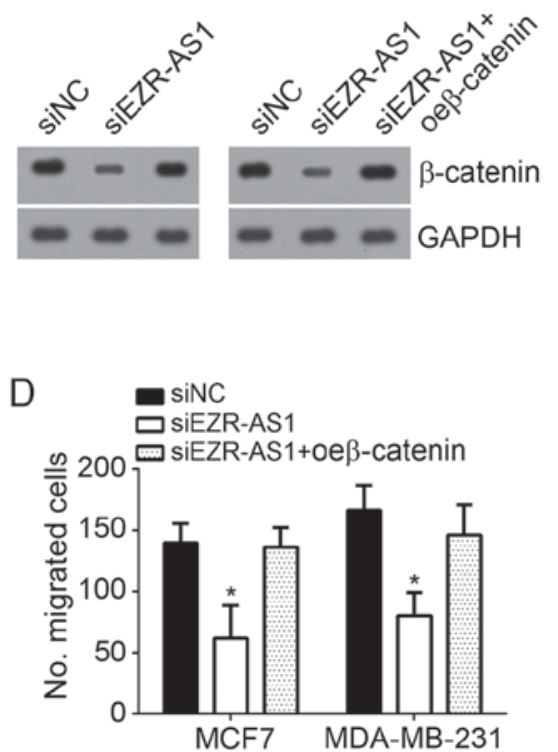

$\mathrm{B}$
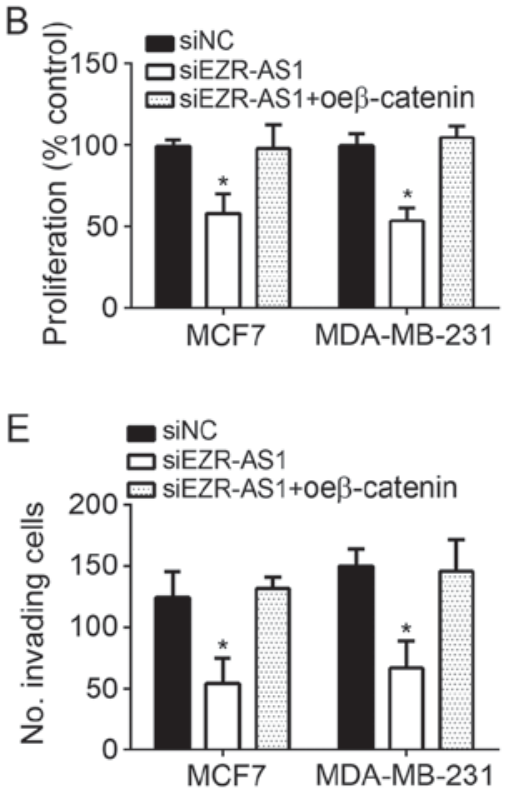

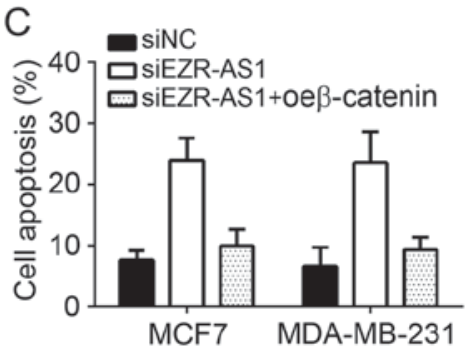

Figure 5. Restoration of $\beta$-catenin expression abrogated the effects of EZR-AS1 knockdown. (A) Western blotting was performed to measure $\beta$-catenin expression in MCF7 and MDA-MB-231 cells. (B) Cell Counting Kit 8 assays were performed to assess cell proliferation. (C) Flow cytometry analysis was utilized to assess apoptosis. (D) Transwell and (E) Matrigel assays were performed to assess migration and invasion, respectively. ${ }^{*} \mathrm{P}<0.05$ vs. siNC. All data are presented as the mean \pm standard deviation of three independent experiments. si, small interfering RNA; NC, negative control; oe, overexpression.

knockdown inhibited cell proliferation compared with negative control cells, whereas $\beta$-catenin overexpression reversed this effect in MCF7 and MDA-MB-231 cells (Fig. 5B). In addition, $\beta$-catenin restoration decreased BC EZR-AS1 knockdown-induced cell apoptosis (Fig. 5C). The results of Transwell and Matrigel assays revealed that $\beta$-catenin restoration significantly promoted the migration and invasion of EZR-AS1-depleted MCF7 and MDA-MB-231 cells (Fig. 5D and E). Taken together, these data suggest that EZR-AS1 regulates the proliferation, migration, invasion and apoptosis of $\mathrm{BC}$ cells via modulating the $\mathrm{Wnt} / \beta$-catenin pathway.

\section{Discussion}

Breast cancer $(\mathrm{BC})$ is one of the most common and malignant cancers that affects among women around the world (1). However, the underlying mechanisms responsible for BC development and progression remain unknown. LncRNAs have been demonstrated to be associated with a number of human cancers, including BC (19). Determining the function and molecular mechanism of lncRNAs in BC progression is crucial for the development of effective diagnostic biomarkers and therapeutic targets for patients with BC. In the present study, the role of EZR-AS1 in BC was investigated. It was demonstrated that EZR-AS1 expression was significantly upregulated in $\mathrm{BC}$ tissues and cell lines. Furthermore, EZR-AS1 expression was negatively correlated with patient outcome and was revealed to serve as an oncogene in BC.

In recent years, IncRNAs have been widely investigated (20-22). A number of reports have indicated that lncRNAs serve important functions in the regulation of biological processes, including proliferation, cell survival and cell mobility (23). LncRNAs are widely involved in tumorigenesis (24) and a number of lncRNAs have been reported to regulate $\mathrm{BC}$ progression $(25,26)$. Li et al $(27)$ reported that lncRNA ANCR regulated EZH2 degradation and attenuated the invasion and metastasis of BC. Huang et al (28) reported that nuclear factor- $\kappa \mathrm{B}$-interacting lncRNA inhibits the migration and invasion of tongue squamous cell carcinoma cells via suppressing epithelial-mesenchymal transition. A previous study indicated that EZR-AS1 expression is associated with human esophageal squamous cell carcinoma (ESCC) (16). The precise role of EZR-AS1 in BC or other cancers requires further study. In the present study EZR-AS1 was demonstrated to be highly expressed in BC tissues compared with adjacent normal tissues. It was also revealed that EZR-AS1 knockdown significantly inhibited proliferation, migration and invasion, while apoptosis was induced. Furthermore, EZR-AS1 knockdown resulted in cell cycle arrest in $G_{0} / G_{1}$ phase. Together, these data suggest that EZR-AS1 serves as an oncogene and contributes to BC malignance. However, in vivo xenograft experiments are required to confirm the role of EZR-AS1 in BC. The results of the present study also suggest that EZR-AS1 may be an effective prognostic marker for patients with BC. However, Cox regression analysis should be performed in future studies to determine whether EZR-AS1 is an independent prognostic factor among other clinicopathological factors.

LncRNAs are able to utilize a diversity of mechanisms to exert their biological functions. Xu et al (29) reported that lncSHRG recruits SATB homeobox 1 to activate Hes family BHLH transcription factor 6 expression and promote hepatocellular carcinoma progression. Shi et al (30) demonstrated that lncHERG acts as a miR-940 sponge to promote proliferation, migration and invasion in glioblastoma. Further reports have revealed that IncRNA breast cancer anti-estrogen resistance 4 (Non-Protein Coding) and lnc- $\beta$-catenin methylation are able to interact with $\beta$-catenin to regulate its stability and 
activate Wnt/ $\beta$-catenin in hepatocellular carcinoma and colon cancer $(31,32)$. These reports indicate that lncRNAs are able to interact with miRNA or proteins to regulate gene expression or protein activity. In the present study, it was demonstrated that EZR-AS1 interacted with $\beta$-catenin in BC cells. Furthermore, it was demonstrated that EZR-AS1 overexpression stabilizes $\beta$-catenin in BC cells, whereas EZR-AS1 knockdown significantly decreased downregulated $\beta$-catenin and activation of the Wnt/ $\beta$-catenin pathway. However, the mechanism by which EZR-AS1 stabilizes $\beta$-catenin requires further investigation

In conclusion, the results of the present study suggest that EZR-AS1 is overexpressed in BC tissues and may serve as a prognostic marker. EZR-AS1 promotes the proliferation, migration and invasion of $\mathrm{BC}$ cells via stabilizing $\beta$-catenin and activating the $\mathrm{Wnt} / \beta$-catenin pathway. Taken together, these results suggest that EZR-AS1 may be a potential therapeutic target for $\mathrm{BC}$ treatment.

\section{Acknowledgements}

Not applicable.

\section{Funding}

No funding was received.

\section{Availability of data and materials}

All data generated or analyzed during this study are included in this published article.

\section{Authors' contributions}

YB and YiW designed this work, performed experiments, analyzed and interpreted the results. YiW initiated this work and wrote this manuscript. XZ, LH, YuW and XL performed experiments. All authors read and approved the final manuscript.

\section{Ethics approval and consent to participate}

Experimental protocols were approved by the Institutional Ethics Committee of Chongqing University Cancer Hospital \& Chongqing Cancer Institute \& Hospital (Chongqing, China) and all patients provided written informed consent.

\section{Patient consent for publications}

Not applicable.

\section{Competing interests}

The authors declare that they have no competing interests.

\section{References}

1. Runowicz CD, Leach CR, Henry NL, Henry KS, Mackey HT, Cowens-Alvarado RL, Cannady RS, Pratt-Chapman ML, Edge SB, Jacobs LA, et al: American Cancer Society/American Society of clinical oncology breast cancer survivorship care guideline. J Clin Oncol 34: 611-635, 2016.
2. Hong W and Dong E: The past, present and future of breast cancer research in China. Cancer Lett 351: 1-5, 2014.

3. Zhang Z, Sun L, Zhang Y,Lu G,Li Y and Wei Z: Long non-coding RNA FEZF1-AS1 promotes breast cancer stemness and tumorigenesis via targeting miR-30a/Nanog axis. J Cell Physiol, 2018.

4. Sun Y, Zeng C, Gan S, Li H, Cheng Y, Chen D, Li R and Zhu W: LncRNA HOTTIP-mediated HOXA11 expression promotes cell growth, migration and inhibits cell apoptosis in breast cancer. Int J Mol Sci 19: E472, 2018

5. Li Y, Jiang B, Wu X, Huang Q, Chen W, Zhu H, Qu X, Xie L, Ma X and Huang G: Long non-coding RNA MIAT is estrogen-responsive and promotes estrogen-induced proliferation in ER-positive breast cancer cells. Biochem Biophys Res Commun: S0006-291X(18)31215-4, 2018.

6. Shi D, Zhang Y, Lu R and Zhang Y: The long non-coding RNA MALAT1 interacted with miR-218 modulates choriocarcinoma growth by targeting Fbxw8. Biomed Pharmacother 97: 543-550, 2018.

7. Ye B, Liu B, Yang L, Zhu X, Zhang D, Wu W, Zhu P, Wang Y, Wang S, Xia P, et al: LncKdm2b controls self-renewal of embryonic stem cells via activating expression of transcription factor Zbtb3. EMBO J 37: e97174, 2018.

8. Caldwell KK, Hafez A, Solomon E, Cunningham M and Allan AM: Arsenic exposure during embryonic development alters the expression of the long noncoding RNA growth arrest specific-5 (Gas5) in a sex-dependent manner. Neurotoxicol Teratol 66: 102-112, 2018

9. Liu B, Ye B, Yang L, Zhu X, Huang G, Zhu P, Du Y, Wu J, Qin X, Chen R, et al: Long noncoding RNA $1 n c K d m 2 b$ is required for ILC3 maintenance by initiation of Zfp292 expression. Nat Immunol 18: 499-508, 2017.

10. Zhu P, Wang Y, Wu J, Huang G, Liu B, Ye B, Du Y, Gao G, Tian Y, He L and Fan Z: LncBRM initiates YAP1 signalling activation to drive self-renewal of liver cancer stem cells. Nat Commun 7: 13608, 2016.

11. Luo Y, Ouyang J, Zhou D, Zhong S, Wen M, Ou W, Yu H, Jia L and Huang Y: Long noncoding RNA GAPLINC promotes cells migration and invasion in colorectal cancer cell by regulating miR-34a/c-MET signal pathway. Dig Dis Sci 63: 890-899, 2018.

12. Chen C, Zhou L, Wang H, Chen J, Li W, Liu W, Shen M, Liu H and $\mathrm{Fu} \mathrm{X}$ : Long noncoding RNA CNALPTC1 promotes cell proliferation and migration of papillary thyroid cancer via sponging miR-30 family. Am J Cancer Res 8: 192-206, 2018.

13. Wang Y, Zhang W, Wang Y and Wang S: HOXD-AS1 promotes cell proliferation, migration and invasion through miR-608/FZD4 axis in ovarian cancer. Am J Cancer Res 8: 170-182, 2018.

14. Pan L, Liang W, Gu J, Zang X, Huang Z, Shi H, Chen J, Fu M, Zhang P, Xiao X, et al: Long noncoding RNA DANCR is activated by SALL4 and promotes the proliferation and invasion of gastric cancer cells. Oncotarget 9: 1915-1930, 2018.

15. Tang R, Wu JC, Zheng LM, Li ZR, Zhou KL, Zhang ZS, Xu DF and Chen C: Long noncoding RNA RUSC1-AS-N indicates poor prognosis and increases cell viability in hepatocellular carcinoma. Eur Rev Med Pharmacol Sci 22: 388-396, 2018.

16. Zhang XD, Huang GW, Xie YH, He JZ, Guo JC, Xu XE, Liao LD, Xie YM, Song YM, Li EM and Xu LY: The interaction of lncRNA EZR-AS1 with SMYD3 maintains overexpression of EZR in ESCC cells. Nucleic Acids Res 46: 1793-1809, 2018.

17. Livak KJ and Schmittgen TD: Analysis of relative gene expression data using real-time quantitative PCR and the 2(-Delta Delta C(T)) method. Methods 25: 402-408, 2001.

18. Luo X, Qiu Y, Jiang Y, Chen F, Jiang L, Zhou Y, Dan H, Zeng X, Lei YL and Chen Q: Long non-coding RNA implicated in the invasion and metastasis of head and neck cancer: Possible function and mechanisms. Mol Cancer 17: 14, 2018.

19. Zhou S, Wang L, Yang Q, Liu H, Meng Q, Jiang L, Wang S and Jiang W: Systematical analysis of IncRNA-mRNA competing endogenous RNA network in breast cancer subtypes. Breast Cancer Res Treat 169: 267-275, 2018.

20. Zhang YX, Yuan J, Gao ZM and Zhang ZG: LncRNA TUC338 promotes invasion of lung cancer by activating MAPK pathway. Eur Rev Med Pharmacol Sci 22: 443-449, 2018.

21. Peng R, Luo C, Guo Q, Cao J, Yang Q, Dong K, Wang S, Wang K and Song C: Association analyses of genetic variants in long non-coding RNA MALAT1 with breast cancer susceptibility and mRNA expression of MALAT1 in Chinese Han population. Gene 642: 241-248, 2018.

22. Liu G, Hu X and Zhou G: Long non-coding RNA OR3A4 promotes proliferation and migration in breast cancer. Biomed Pharmacother 96: 426-433, 2017. 
23. Lai XJ and Cheng HF: LncRNA colon cancer-associated transcript 1 (CCAT1) promotes proliferation and metastasis of ovarian cancer via miR-1290. Eur Rev Med Pharmacol Sci 22: 322-328, 2018.

24. Xu W, He L, Li Y, Tan Y, Zhang F and Xu H: Silencing of IncRNA ZFAS1 inhibits malignancies by blocking Wnt/ $\beta$-catenin signaling in gastric cancer cells. Biosci Biotechnol Biochem 82: 456-465, 2018

25. Zhang $\mathrm{P}$, Zhou $\mathrm{H}$, Lu K, Lu Y, Wang $\mathrm{Y}$ and Feng $\mathrm{T}$ : Exosome-mediated delivery of MALAT1 induces cell proliferation in breast cancer. Onco Targets Ther 11: 291-299, 2018.

26. Zhang S, Wang J, Ghoshal T, Wilkins D, Mo YY, Chen Y and Zhou Y: IncRNA gene signatures for prediction of breast cancer intrinsic subtypes and prognosis. Genes (Basel) 9: E65, 2018

27. Li Z, Hou P, Fan D, Dong M, Ma M, Li H, Yao R, Li Y, Wang G, Geng P, et al: The degradation of EZH2 mediated by lncRNA ANCR attenuated the invasion and metastasis of breast cancer. Cell Death Differ 24: 59-71, 2017.

28. Huang W, Cui X, Chen J, Feng Y, Song E, Li J and Liu Y: Long non-coding RNA NKILA inhibits migration and invasion of tongue squamous cell carcinoma cells via suppressing epithelial-mesenchymal transition. Oncotarget 7: 62520-62532, 2016.
29. Xu YC, Liang CJ, Zhang DX, Li GQ, Gao X, Fu JZ, Xia F, Ji JJ, Zhang LJ, Li GM and Wu JX: LncSHRG promotes hepatocellular carcinoma progression by activating HES6. Oncotarget 8: 70630-70641, 2017.

30. Shi J, Wang YJ, Sun CR, Qin B, Zhang Y and Chen G: Long noncoding RNA lncHERG promotes cell proliferation, migration and invasion in glioblastoma. Oncotarget 8: 108031-108041, 2017.

31. Zhu P, Wang Y, Huang G, Ye B, Liu B, Wu J, Du Y, He L and Fan Z: lnc- $\beta$-Catm elicits EZH2-dependent $\beta$-catenin stabilization and sustains liver CSC self-renewal. Nat Struct Mol Biol 23: 631-639, 2016.

32. Ouyang S, Zheng X, Zhou X, Chen Z, Yang X and Xie M: LncRNA BCAR4 promotes colon cancer progression via activating Wnt/ $\beta$-catenin signaling. Oncotarget 8: 92815-92826, 2017. International (CC BY-NC-ND 4.0) License. 